\title{
PEDRO GUTIÉRREZ BUENO (1745-1822) Y LAS RELACIONES ENTRE LA QUÍMICA Y LA FARMACIA DURANTE EL ÚLTIMO TERCIO DEL SIGLO XVIII*
}

\author{
por \\ JoSÉ R. BERTOMEU SÁNCHEZ \\ Universitat de València, CSIC
}

\section{ANTONIO GARCía BELMAR}

Universidad de Alicante

RESUMEN: El presente trabajo se enmarca en un proyecto de investigación sobre la farmacología en la sociedad española del siglo XIX, en particular, acerca del papel de las prácticas y los conocimientos químicos en la transición de la materia médica a la farmacología experimental. Dentro de ese esquema general, el objeto principal de este trabajo es uno de los principales autores españoles de libros de texto de química del último tercio del siglo XVIII: Pedro Gutiérrez Bueno (1745-1822). En un trabajo anterior se ba estudiado el público destinatario de la primera edición de su libro de texto así como las principales características de esta obra. En este artículo abordaremos el análisis del contexto en el que se produjo la aparición de la segunda edición, lo que conducirá a estudiar las ideas de Gutiérrez Bueno sobre las relaciones entre la química y la farmacia. Estas ideas estuvieron fuertemente influidas por los puntos de vista defendidos por Antoine Fourcroy en Francia.

Palabras clave: Historia de la química. Revolución química. Públicos de la ciencia. Pedro Gutiérrez Bueno (17451822). Antoine Fourcroy (1755, 1809).

- El presente artículo forma parte del proyecto de investigación «La transformación de la materia médica en terapéutica farmacológica en la España del siglo XIX» (PB96-0761-C03-03). Queremos manifestar nuestro agradecimiento al profesor Francisco J. Puerto Sarmiento y al resto de miembros del Departamento de Historia de la Farmacia de la Universidad Complutense de Madrid que nos han ayudado en la consulta de la documentación del Colegio de Farmacia de Madrid conservada en este departamento.

Hispania, LXI/2, núm. 208 (2001) 539-562 
ABSTRACT: This paper is part of a general research project on the role that chemistry played in transforming experimental pharmacology during nineteenth-century Spain. Within this general framework, this paper deals with the main characteristics of chemistry textbooks written by Pedro Gutiérrez Bueno (1745-1822), a pharmacist who taught chemistry in several institutions during the late eighteenth century. In an earlier paper, we bave analysed the intended public of the first edition of Gutierrez Bueno's textbook. This paper deals with the changing institutional context in which the second edition was published. This issue is closely connected with substantial transformations in the relationship between chemistry and pharmacy that took place during this period. Therefore, Gutierrez Bueno's ideas about this problem are examined as well as his major role in appropriating some of Antoine Fourcroy's views on chemistry and pharmacy.

KEY WORDS: History of chemistry. Chemical Revolution. Audiences of chemistry. Pedro Gutiérrez Bueno (1745-1822). Antoine Fourcroy (1755-1809).

Pedro Gutiérrez Bueno (1745-1822) publicó entre 1788 y 1803 lo que pueden considerarse como dos ediciones de un mismo libro de texto de química. La primera edición estaba dirigida a los estudiantes que asistían a las clases que impartió en el Real Laboratorio de Química de Madrid durante las últimas décadas del siglo XVIII. La segunda fue inicialmente publicada para los estudiantes del Colegio de Cirugía de Madrid pero, probablemente, fue también empleada en sus cursos de química en el Colegio de Farmacia durante los primeros años del siglo XIX ${ }^{1}$.

En un trabajo reciente, se han analizado las características generales de la primera edición del curso de Gutiérrez Bueno y de su público destinatario. Entre otras cuestiones, se ha mostrado que varios grupos de oyentes asistieron al curso del Real Laboratorio de Química de Madrid. El primero estaba formado por el público habitual de las lecciones de química: médicos y farmacéuticos que buscaban en estas clases un complemento a su formación profesional. El segundo grupo era, por el contrario, un buen representante de los nuevos públicos de la química surgidos durante el siglo XVIII. Se trataba de nobles o comerciantes adinerados, que veían la química como un pasatiempo intelectual o un complemento a la educación general del hombre ilustrado. El interés que

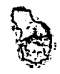

1 De la primera edición apareció anónimamente sólo un volumen: [GUTIERREZ BUENO, P.] (1788) Curso de química, teórico y práctica, para la enseñanza del Real Laboratorio de Química de esta Corte. Madrid, Antonio Sancha, $250+72$ p. De la segunda edición aparecieron dos volúmenes, aunque se anunció un tercero que debía ampliar la parte práctica: Curso de química, dividido en lecciones para la enseñanza del Real Colegio de San Carlos. Por D... Madrid, Villalpando, 1802, 1 h. + 294 p; Práctica del Curso de Química dividido en lecciones para la enseñanza del Real Colegio de San Carlos. Madrid, Villalpando, 1803. 304 p. Su versión de la nueva terminología química apareció en 1788 y fue reeditada a principios del siglo XIX: Nomenclatura química, que para el uso de su escuela pública... Segunda edición; más cómoda para los profesores de las tres facultades del arte de curar. Madrid, Sancha, 1801. 16 h. +123 p. 
Gutiérrez Bueno prestó a asuntos relacionados con los tintes y el blanqueado de telas sugiere que también debieron asistir a sus clases algunos artesanos. De este modo, al planificar sus clases y al redactar su obra, Gutiérrez Bueno tuvo que afrontar la tensión entre los diferentes intereses de este heterogéneo público. La estrategia adoptada por Gutiérrez Bueno, de acuerdo con sus palabras, consistió en la ampliación de la química «en las teorías y en sus aplicaciones». La parte teórica de la química fue aumentada con la creación de capítulos acerca de la naturaleza del fuego, el aire, el agua y las tierras que constituyeron la mayor parte del único volumen publicado de su libro de texto. A las «aplicaciones de la química», Gutiérrez Bueno consagró buena parte de sus discursos de apertura y de la introducción de su libro de texto así como diversos escritos dedicados a su empleo en medicina y en la industria ${ }^{2}$.

La situación en la que se produjo la aparición de la segunda edición del curso de química de Gutiérrez Bueno fue bastante diferente. Su nuevo público eran los estudiantes del Colegio de Cirugía de San Carlos de Madrid, una institución que, en esos años, estaba sufriendo diversos cambios como consecuencia de las reformas de la enseñanza y del ejercicio de la medicina, la cirugía y la farmacia. Más adelante, Gutiérrez Bueno pasó a ocupar una cátedra en el Colegio de Farmacia de Madrid, donde desarrolló sus últimos años de enseñanza hasta su jubilación en 1815 .

Este trabajo pretende analizar las características generales del nuevo marco en el que se produjo la enseñanza de la química durante los años de transición del siglo XVIII al XIX. En primer lugar, se estudiará el nuevo marco institucional donde se insertaron las clases de Gutiérrez Bueno, con especial atención al papel otorgado a la química en estas nuevas instituciones educativas. Este análisis conducirá a analizar la asimilación en España de las ideas de Antoine Fourcroy acerca de las relaciones entre la química y la farmacia. Como se verá, Gutiérrez Bueno y sus discípulos jugaron un papel decisivo en la introducción de estas ideas, por lo que se hace necesario analizar, en primer lugar, las propuestas de este autor francés para pasar, a continuación, al estudio de los puntos de vista de Gutiérrez Bueno.

2 Garcia Belmar, A.; Bertomeu Sanchez, J. R.: «Pedro Gutiérrez Bueno (1745-1822), los libros de texto y los nuevos públicos de la química en el último tercio del rsiglo XVIII», Dynamis (en publicación). El marco institucional ha sido discutido en BERTOMEU SANCHEZ, J. R. GARCIA BELMAR, A.: «Los libros de texto de enseñanza de la química destinados a estudiantes de medicina y cirugía en España (1788-1845)», Dynamis,20, 457-489. Un panorama más general junto con la discusión de los planteamientos generales la hemos ofrecido en BERTOMEU SANCHEZ, J.R.; GARCIA BELMAR, A.: «Spanish chemistry textbooks (1800-1845). A bibliographical approach» y GARCIA Belmar, A.; Bertomeu SANCHEZ, J.R.: «French chemistry textbooks (1802-1852). New books for new readers and new teaching institutions». En: B. BENSAUDE-VINCENT; A. LUNDGREN (eds.): Comunicating Chemistry: Textbooks and their Audiences, 1789-1939, Canton, History of Science Publications, 2000, pp. 40-91. En estos trabajos se ofrece orientación bibliográfica sobre algunos de los temas tratados en este artículo.

Hispania, LXI/2, núm. 208 (2001) 539-562 


\section{El NUEVo Marco INSTITUCiONAL DE la ENSEÑANZA DE LA QUímiCa}

Al contrario que la primera, la segunda edición del curso de Gutiérrez Bueno fue escrita para un grupo muy homogéneo de estudiantes: aquéllos que asistían a las lecciones del Colegio de Cirugía de San Carlos. La nueva situación fue propiciada por uno de los primeros intentos de reunión de la medicina y la cirugía que tuvo lugar a principios del siglo XIX en España. Como consecuencia de esta reforma, que tuvo una corta duración, el Colegio de Cirugía de Madrid se unió al Real Estudio de Medicina Práctica y pasó a ser regido por una «Junta General de Gobierno de la Facultad reunida». Tales cambios afectaron también a los estudios y al ejercicio de la farmacia. La Concordia y Reales Ordenanzas de 1800 establecían que los boticarios debían obtener el título de bachiller en artes y estudiar dos años en «cualquiera de los reales colegios de la facultad reunida», lo que les permitía obtener el título de «bachiller en química». Para poder regentar una botica era necesario que, además de estos estudios teóricos, se realizaran dos años de aprendizaje, junto a un boticario aprobado, lo que conducía a la obtención del título de licenciado en farmacia después de superar el correspondiente examen. Estas ordenanzas preveían, además, un título de «doctor en química» como "grado de pompa y honor». La nueva situación tuvo muy corta vigencia puesto que la «facultad reunida» fue suprimida en abril de $1801 \mathrm{y}$, tras unos años de diversas reformas, una nueva legislación estableció en 1804 las Juntas de Gobierno de la Medicina, la Cirugía y la Farmacia ${ }^{3}$.

Gutiérrez Bueno fue nombrado en 1801 catedrático de química del Colegio de Cirugía de Madrid y ocupó este cargo hasta 1804, cuando, junto con su ayudante, José Meneses, fue apartado del Colegio debido a la aparición de nuevas ordenanzas. De acuerdo con la nueva legislación, la enseñanza de este Colegio debía «ser de pura Cirugía» y debían separarse del mismo «los Médicos y Farmacéuticos que estaban empleados» ${ }^{4}$. Durante esos breves tres años de enseñanza en el Colegio de San Carlos de Madrid, Pedro Gutiérrez Bueno publicó un libro de texto de química en dos volúmenes. Según su testimonio, pretendía evitar a sus alumnos la copia de apuntes de sus clases y facilitarles la lectura de los contenidos de sus lecciones antes de asistir a las mismas:

3 Concordia y Reales Ordenanzas para el régimen y gobierno de la facultad de Farmacia, 1800. Artículos 2-5. Se encuentra reproducido en Chiarlone, Q.; Mallaine, C., Historia de la Farmacia ..., Madrid, José M. Ducazcal, 1865, pp. 790-808. Sobre los resultados de estas reformas véase, por ejemplo, la obra de Aparicio Simón, J. , Historia del Real Colegio de San Carlos de Madrid, Madrid, Aguilar, 1956.

4 Archivo de la Universidad Complutense (AUC), Legajo D-395, Orden el Ministro de Gracia y Justicia comunicada al Colegio de Cirugía de Madrid, 27 de abril de 1804. No obstante, las nuevas ordenanzas, preveían la existencia de una cátedra de «materia médica, botánica, química y farmacia médica» que dirigió Manuel Bonafós. Aparicio Simón destaca «los lucidos exámenes de Química y la verdadera ceremonia con que se celebraron en el Colegio" bajo la presidencia de Pedro Gutiérrez Bueno durante el curso 1801-1802. V. Aparicio Simón (1956),op. cit. (3), pp. 109-112.

Hispania, LXI/2, núm. 208 (2001) 539-562 
«Por esto ha parecido muy del caso irles repartiendo impresas las lecciones de una para otra, para que quando vengan a la Cátedra traigan ya leída la lección que se les va a dar, y comprehendan mejor la explicación, la satisfacción a las dudas que les ocurran, y la demostración que se haga mediante los aparatos químicos. Por este medio es de esperar que adelanten mucho más que oyendo sólo la viva voz del maestro, con tal que estudien con mucha atención cada una de las lecciones principalemnte las que tratan de las sustancias simples y de las propiedades naturales de los cuerpos, no pasando a la segunda sin haber entendido bien la primera»s.

Según su testimonio, la obra había sido escrita «con bastante apresuración» debido a que intentó ofrecerla "por pliegos» a sus estudiantes "pocos días antes» del comienzo de las enseñanzas ${ }^{6}$. También para estas clases, Gutiérrez Bueno publicó la segunda edición de la nueva nomenclatura química ${ }^{7}$. Probablemente estas obras fueron empleadas en sus cursos impartidos en el Colegio de Farmacia de Madrid. En las ordenanzas de 1804, que regulaban el estudio y el ejercicio de la farmacia, se establecía que los estudiantes debían matricularse en los colegios de farmacia, para lo que debían presentar certificados de limpieza de sangre y de haber estudiado «la gramática latina, la lógica y las matemáticas", consideradas como "absolutamente necesarias para la debida comprensión de la química» ${ }^{8}$. Para ello, el Colegio estableció exámenes públicos de estas materias que permitían a los que lo superaban la obtención del título de Bachiller en Artes9. Una vez superados estos requisitos debían estudiar tres años en los colegios de farmacia, de acuerdo con el siguiente plan de estudio:

5 GUTIERreZ BUENO (1802), op. cit. (1), pp. 5-6.

6 Ibid. p. 262: «Aquí se dará fin a las lecciones que se han de dar en esta escuela en dos cursos. En ellas disimularán los oyentes las faltas que hayan podido notar en una obra escrita con bastante apresuración, por haberse determinado su impresión, para entregarla por pliegos a los que asisten a la enseñanza, pocos días antes de que esta comenzase.»

7 Gutierrez Bueno, P.: Nomenclatura química, que para el uso de su escuela pública... Segunda edición; más cómoda para los profesores de las tres facultades del arte de curar. Madrid, Sancha, $1801.16 \mathrm{~h}$. $+123 \mathrm{p}$.

8 REAL CEDULA ... por la cual se aprueban y mandan observar las nuevas ordenanzas para el régimen y gobierno de la facultad de farmacia..., Madrid, Imprenta Real, 1804, cap. III, art. 16.

9 Folch Jou, G.: Historia del Real Colegio de San Fernando, Madrid, 1977, pp. 39 y 44 . Además de este trabajo, para una información más actualizada sobre la historia de la enseñanza de la farmacia en el período estudiado, pueden consultarse los diferentes estudios de Rafael Roldán Guerrero, Ramón Gago Bohorquez, Javier Puerto Sarmiento y la excelente tesis de Carmen Calleja Folguera, parte de la cual ha aparecido publicada en diversos artículos, que contiene abundante información de archivo. Las referencias bibliográficas de estos trabajos se encuentran en nuestros estudios citados en la nota. Para obtener información bibliográfica actualizada puede consultarse el repertorio que anualmente publica la revista Asclepio con el título de Bibliografía Histórica sobre la Ciencia y la Técnica en España. 
«en el primero oirán las que debe dar el Catedrático de Historia Natural: en el segundo el que explique el Catedrático de Química, asistiendo de nuevo a las de Historia Natural; en el tercero repetirán el curso de Química»10.

La asistencia a estos cursos era obligatoria para los estudiantes del Colegio, tras lo cual era posible aspirar al título de bachiller en farmacia una vez superado el correspondiente examen. Al igual que en las normas anteriores, estos estudios académicos debían ser complementados con el tradicional aprendizaje en las boticas, aunque se preveía la posibilidad de desarrollar esta formación práctica en los colegios. En la convocatoria de las clases del otoño de 1806, se indicaba que, para ser admitidos a examen, los practicantes de farmacia debían presentar "certificación de haber sido matriculados en la escuela y de haber asistido a todas las lecciones por el tiempo que les falte de edad hasta cumplir los 25 años» ${ }^{11}$. Los mayores de veinticinco años y los que trabajaban en hospitales militares estaban exentos de la asistencia a las clases del Colegio ${ }^{12}$. En cualquier caso, las ordenanzas de 1804 establecían que, para poder regentar botica, era necesario obtener el título de licenciado que suponía realizar dos años de práctica «en los laboratorios de los mismos colegios» o «con boticarios aprobados» y la superación de un examen dividido en una parte teórica y otra práctica. En la primera, el candidato podía ser interrogado acerca de las materias estudiadas en el colegio y en la segunda debía realizar «dos operaciones químico-farmacéuticas» y describir varias plantas «según el sistema de Linneo» $\mathrm{y}$ «algunas producciones de los reinos animal y mineral» ${ }^{13}$.

Las enseñanzas debían ser impartidas por dos catedráticos junto con sus respectivos ayudantes. Uno de ellos debía ofrecer lecciones de «historia natural en sus tres reinos" y el otro de "química y farmacia». Gutiérrez Bueno se incorporó con cierto retraso a su puesto como profesor de esta última materia debido a una enfermedad. Según la descripción ofrecida por sus estudiantes, durante su primera clase, Gutiérrez Bueno leyó «algo balbuciente [...] un discurso en voz baja [...] que no se le entendía bien [...] por el ruido de la gente que entraba y salía, por la voz baja con que lo pronunciaba [...] por tener la boca ocupada con unas hojas [...] por que, según decía el mismo Bueno, se hallaba constipado». También observó un estudiante que Gutiérrez Bueno «sólo daba

10 Real Cedula (1804), op. cit. (8), Capítulo III, Artículo 18

11 Archivo del Museo de Historia de la Farmacia (AMHF), Madrid, Libro de acuerdos del Colegio de Farmacia, f. 31.

12 FOLCH JOU (1977), op. cit. (9), p. 40. Numerosos ejemplos de estas situaciones se encuentran en las peticiones formuladas al Colegio: Cf. AMHF, Libro de Acuerdos del Real Colegio de Farmacia de Madrid.

13 ReAl Cedula (1804), op. cit. (8). Cap. IV, artículos 1-6. Sobre el número de estudiantes, que varió entre setenta durante el primer año hasta dieciseis en 1816, v. FOLCH JOU, G.: (1977), op. cit. (9), pp. 41-42.

Hispania, LXI/2, núm. 208 (2001) 539-562 
movimiento a uno de los dos brazos» ${ }^{14}$. Durante los años siguientes, Gutiérrez Bueno sufrió problemas de salud que le impidieron impartir muchas de sus clases hasta que, finalmente, se vio obligado a solicitar la jubilación en 1815.

La nueva forma de enseñanza tuvo que enfrentarse a ciertas resistencias de los partidarios de la formación tradicional basada en el aprendizaje realizado en las boticas. Con la creación de los colegios, los boticarios aprobados no sólo perdían protagonismo en el sistema formativo sino que se veían obligados a prescindir de la colaboración de sus ayudantes durante las horas de clases. Poco después de constituida la nueva institución, en junio de 1806, los profesores manifestaban que habían tenido noticia de estas actitudes entre los boticarios de la corte:

«algunos Boticarios de esta Corte obstinados en contrarrestar del modo que les es posible las Reales Ordenanzas de S.M relativas a la asistencia de sus practicantes a esta Escuela, retienen en sus casas algunos, que habiéndose desentendido de aquellas Reales ordenes permanecen sin haberse matriculado, ni asistir a esta Escuela, persuadidos por sus maestros a que de esta omisión ningún perjuicio les ha de resultar ...»15

El Colegio solicitó una nómina de los practicantes existentes en las boticas de Madrid pero el problema persistió en los años siguientes y quizás fue una de las causas de las dificultades financieras de los primeros años del Colegio. Aunque Gutiérrez Bueno, como catedrático de mayor edad, fue nombrado «jefe local» del Colegio, la dirección del mismo, tanto «en lo económico como en lo literario», quedaba en manos de la Junta de Farmacia, que era la encargada de gestionar los sustanciosos ingresos que producían la expedición de títulos, la visita de boticas y la venta de obras como la Farmacopea Hispana y la tarifa y el petitorio farmacéutico. Esta situación produjo fuertes tensiones entre la Junta y los profesores ${ }^{16}$. Varios años después de su nombramiento como catedrático, Gutiérrez Bueno se quejaba del escaso apoyo recibido de la Junta para mantener el jardín botánico y el laboratorio de química previsto por la Real Cédula de 1804:

«habiendo escatimado la Junta [de Farmacia] desde su principio los auxilios no han sido suficientes ... para mantener y aumentar la Biblioteca que no deja sin embargo de estar surtida; también está bien provisto el Gabinete de mineralogía aunque no completo. El de Zoología apenas tiene un exemplar y el herbario está muy escaso.

14 AMHF, Libro de acuerdos del Colegio de Farmacia, f. 41 v. Junta ordinaria de 8 de octubre de 1806.

15 AMHF, Libro de acuerdos del Colegio de Farmacia, 10v., Junta ordinaria de 18 de junio de 1806.

16 Sobre las atribuciones de la Junta, v. Ordenanzas... 1804, capítulo III, Artículo 3. Acerca de la polémica entre el Colegio y Gutiérrez Bueno, v. BerTomeu SANCHEZ, J. R.: «La censura gubernativa de libros científicos durante el reinado de José I en España (1808-1813)", Hispania, 54 (188) (1994), 917-954 y BERTOMEU SANCHEZ, J.R.: La actividad científica en España bajo el reinado de Jose I (1808-1813)..., Valencia, Universitat de València, 1995 pp. 99-102.

Hispania, LXI/2, núm. 208 (2001) 539-562 


\begin{abstract}
Asimismo tiene el Colegio su Jardín muy pequeño y su Laboratorio falto de instrumentos; la Casa en que están uno y otro no es propia ...»17
\end{abstract}

A todos los profesores se les exigía escribir un «curso elemental» de su asignatura para que les sirviera «en sus explicaciones, y a los discípulos de texto». La obra debía redactarse «en el estilo más correcto, claro y sucinto posible, pero comprendiendo todas las doctrinas conducentes a la instrucción de sus alumnos» ${ }^{18}$. En el caso de la química, se señalaba que, hasta la publicación del curso previsto, debía adoptarse el Tratado elemental de química de Lavoisier aplicando tales conocimientos al «arte de curar»:

«el [...] catedrático, que ha de enseñar la química y la farmacia» [...] [deberá hacerlo] valiéndose, entre tanto que se publica el curso prevenido en el artículo VII, de los elementos de Lavoisier, haciendo de ellos las aplicaciones en teórica y práctica a las operaciones que tienen relación con el arte de curar, y dando a conocer a sus discípulos las sustancias elementales o simples según el estado actual de los conocimientos químicos, como son la luz, el calórico, los gases, y los resultados de sus mezclas y combinaciones entre sí, y en los demás cuerpos de las bases acidificables y su oxigenación; de los principios de los animales y vegetales; alteraciones que padecen unos y otros por el fuego, por la fermentación y putrefacción, confirmando las teorías con experiencias o ejemplos que tengan relación con las operaciones farmacéuticas, concluyendo por la farmacia» 19

Los contenidos y estructura del curso publicado por Pedro Gutiérrez Bueno en 1802 se ajustaban perfectamente a los temas exigidos en el párrafo anterior, lo que hace suponer que fue empleado en las clases que impartió entre 1806 y 1815. A pesar de sus problemas de salud, Gutiérrez Bueno siguió trabajando en la elaboración de nuevas obras de enseñanza. En noviembre de 1806, urgió al resto de sus compañeros «a tratar de componer los elementos que con arreglo a la Real Ordenanza» debían "servir de texto en la enseñanza de la escuela» ${ }^{20}$. A finales de 1808 , presentó para su censura «una obra de química en 32 cuadernos» que no parece que llegara a publicarse ${ }^{21}$. Es posible que fuera una versión previa de su «Prontuario de Química, Materia Médica y Farmacia» que apareció en 1815. La obra estaba destinada — según su autor- a «los facultativos de la Ciencia de Curar» que "por necesidad o por falta de medios» no hubieran "podido cursar las Ciencias auxîliares, como la Química y Farmacia». Se trataba de un pequeño manual escrito en forma de preguntas y repuestas

17 Archivo Municipal de Madrid, Secretaría, Legajo 2-353-25. Contestación de Pedro Gutiérrez Bueno y Antonio de la Cruz al Prefecto de Madrid. Madrid, 25 de enero de 1811.

18 REAL Cedula (1804), op. cit. (8), capítulo III, artículo 7.

19 Ibid. Capítulo III, artículo X.

20 AMHF, Libro de acuerdos del Colegio de Farmacia, f. 53, Junta ordinaria de 12 de noviembre de 1806.

21 Ibid. f. 144, junta de 25 de noviembre de 1808.

Hispania, LXI/2, núm. 208 (2001) 539-562 
con el objetivo de hacer «fácil de dar a la memoria» los conocimientos de química, farmacia e historia natural útiles para los jóvenes que se dedicaran «al arte de curar y a la Química» ${ }^{22}$.

En definitiva, tanto la segunda edición de su curso como este pequeño prontuario estaban dirigidas a estudiantes de medicina, cirugía y farmacia en unos años de intensos cambios en el estudio y el ejercicio de estas profesiones. A lo largo del siglo XVIII, la farmacia y la cirugía se desprendieron del control de los gremios para pasar bajo la jurisdicción real del tribunal del protomedicato $\mathrm{y}$, más adelante, de las juntas creadas a principios del siglo XIX que lo sustituyeron. En el caso de la farmacia, estas transformaciones también supusieron el abandono de la tradicional enseñanza práctica realizada en las boticas, a través de los años de aprendizaje, para dejar paso, poco a poco, a una formación académica realizada en los colegios de farmacia que, a mediados del siglo XIX, quedó integrada dentro de la Universidad ${ }^{23}$.

Muchas de estas transformaciones fueron inspiradas por las reformas que habían tenido lugar en Francia en los años de la Revolución y el Imperio en las que participó un autor que, como veremos, tuvo gran influencia en la obra de Gutiérrez Bueno y de otros químicos españoles: Antoine Fourcroy. Como se verá en el siguiente apartado, tanto el propio Gutiérrez Bueno como algunos de sus primeros discípulos desempeñaron un papel importante en la asimilación de las ideas de Fourcroy en España.

\section{ANTOINE FOURCROY (1755-1809) Y LAS NUEVAS RELACIONES ENTRE LA QUÍMICA Y LA FARMACIA}

Los nombres de los títulos «bachiller» y «doctor en química», mencionados en el apartado anterior, indican la íntima relación — si no identidad- que para muchos farmacéuticos existía entre la química y la farmacia. Esta relación sufrió fuertes cambios durante la segunda mitad del siglo XVIII. Debido a estas transformaciones, que afectaron tanto a los contenidos teóricos como a la

22 Gutierrez Bueno, P.: Prontuario de Química, farmacia y materia médica, dividido en tres secciones. Madrid, Villalpando, 1815, pp. iv-v. Teniendo en cuenta el escaso número de reediciones de textos clásicos científicos españoles, resulta sorprendente que este pequeño texto haya sido reimpreso en dos ocasiones en los últimos años: Prontuario de Química, Farmacia y Materia Médica (prólogo de M.C. Francés), Madrid, Instituto de España / Real Academia de Farmacia, 1994 y id. Valencia, Librerías París-Valencia, 1998. Por desgracia, ninguna de las dos ediciones contiene un estudio crítico de la obra, por lo que resulta necesario recurrir al análisis realizado por FOLCH JOU, G.; SANCHEZ-Moscoso, A.: «Estudio del «Manual de Química, Farmacia y Materia Médica» de Pedro Gutiérrez Bueno", Boletín de la Sociedad Española de Historia de la Farmacia, 28, (1975) 91-8. Sobre los problemas con la censura de esta obra, v. BerTOMEu SANCHEz (1994), op. cit. (16).

23 Sobre estas cuestiones, v. Puerto Sarmiento, F. J.: «Ciencia y farmacia en la España decimonónica». En: LOPEZ PIÑERO, J. M.: (ed.), La ciencia en la España del siglo XIX, Madrid, Ayer, 1992, pp. 153-193.

Hispania, LXI/2, núm. 208 (2001) 539-562 
posición institucional de la química y de la farmacia, se consolidó la diferenciación entre ambas disciplinas que, a pesar de ello, continuaron manteniendo estrechas relaciones en diversos planos. En su estudio acerca de este proceso en Francia, Jonathan Simon ha señalado la necesidad de tener en cuenta estas transformaciones disciplinares para comprender correctamente algunos aspectos de la revolución química. Según Simon, uno de los principales protagonistas de estos cambios fue Antoine Fourcroy que defendió nuevos puntos de vista sobre las relaciones entre la química y la farmacia a través de varios escritos y los materializó mediante las transformaciones institucionales que impulsó en el marco de la Revolución Francesa ${ }^{24}$.

$\mathrm{La}$ influencia de las ideas de Fourcroy en la química y la farmacia española de esos años resulta patente cuando se analizan tanto las instituciones como los libros publicados durante el período estudiado. Los Colegios de Farmacia creados en 1804 estaban claramente inspirados en las Ecoles de Pharmacie establecidas un año antes. Las disciplinas enseñadas en las dos instituciones eran bastante similares (historia natural, química y farmacia) y, aunque con diferentes requisitos, las dos legislaciones limitaban el papel de los gremios en el control del ejercicio de la farmacia y establecían una enseñanza académica junto a la tradicional formación práctica en las boticas ${ }^{25}$.

Los escritos de Fourcroy tuvieron gran difusión en España a través de traducciones y de obras de autores españoles. La mayor parte de sus libros de texto y el gran tratado de química que escribió fueron traducidos al castellano. Estas obras sirvieron como fuentes de información y como modelo para muchos libros de texto de química escritos por autores españoles hasta las primeras décadas del siglo XIX. Algunos de los pensionados en París asistieron a sus cursos, lo que les permitió asimilar muchas de sus ideas de primera mano. Entre todos ellos destaca Juan Manuel de Aréjula (1755-1830) quien, a su vuelta de la capital francesa, se hizo cargo de la enseñanza de la química en el Colegio de Cirugía de Cádiz, desde donde defendió posturas similares a las de Antoine Fourcroy respecto a la relación entre la química y la medicina ${ }^{26}$.

24 SimON, J.: (1998), "The Chemical Revolution and Pharmacy: A Disciplinary Perspective», Ambix, 45 (1), 1-13 que contiene un resumen de parte de su tesis doctoral: The Alchemy of Identity: Pharmacy and Chemical Revolution (1777-1809), University of Pittsburg, Ph. D., 1997, donde trata, con mayor detalle, en las pp. 150-167, el significado de las reformas institucionales.

25 Beauchamp, A.: Recueil des lois et réglements sur l'enseignement supérieur, Paris, Delalain, 1888, t. XXVIII, 600-606. Loi contenant organisation des écoles de pharmacie, 21 germinal de lan XI (11 avril 1803). Sobre esta cuestión, además de la obra de J. Simon citada en la nota anterior, v. PRÉveT, F.: Histoire de lorganisation sociale en pharmacie, Paris, Recueil Sirey, 1940; DILlemanN, G; Michel, M.E.: «La réception des pharmaciens en France de la Révolution à l'application de la loi du 21 germinal an XI (1791-1813)», Revue d'Historie de la Pharmacie, 260 (1984), 42-69. Una breve y discutible revisión de la enseñanza de la química en estas instituciones ha sido realizada en la tesis de ARNAUD, C.: L'enseignement de la chimie minérale à l'Ecole puis à la faculté de pharmacie de Paris, 1803 à 1946, Paris, Thèse de doctorar, 1986, 106 p.

${ }_{26}$ Sobre las traducciones y la influencia de Fourcroy en los libros de texto de química, v. J.R. BertomeU; GARCIA Belmar, A.: op. cit. (2). 
También fueron traducidos al castellano diversos escritos de Fourcroy sobre las relaciones entre la química y la farmacia. El «discourse sur l'union de la Chimie et la Pharmacie», que Fourcroy leyó en enero de 1797 ante la Société Libre de Pharmaciens de Paris, fue publicado en los Annales de Chimie, revista que Gutiérrez Bueno tenía en su biblioteca. Este escrito fue traducido por Francesc Carbonell i Bravo al castellano y publicado en forma de folleto en $1804^{27}$. Fourcroy defendía la necesidad de establecer nuevos vínculos entre la química y la farmacia, tras las transformaciones ocurridas durante la segunda mitad del siglo XVIII que habían producido «une sorte de divorce» entre ambas disciplinas. Según Fourcroy, tal ruptura se había producido como consecuencia del acercamiento de la química a la física experimental:

«quelques années après le milieu de notre siècle, la chimie, portant son vol plus haut, et faisant une sorte de divorce avec sa première mère, vous paroîtroit quitter les laboratoires de pharmacie, et tarnsportée dans les cabinets de physique expérimentale, abandonner l'utile préparation des médicamens, s'élancer à de plus hautes destinées, et donner aux physiques de nouveaux moyens de concevoir comme d'expliquer les plus grands phénomènes de la nature» 28

A través de estos cambios, la química había llegado a ser «plus physique» y extendido su aplicación «a toutes les branches de l'étude de la nature». Para Fourcroy, la farmacia era uno de los principales campos de aplicación de la nueva ciencia química. Gracias a ello, la farmacia podía evitar las consecuencias del «empyrisme aveugle» y de la «mediocrité routinière». Por ello, Fourcroy consideraba que "aucun art ne peut tirer plus de secours de la chimie, et se perfectionner d'avantage par son propre avancement, que celui de la préparation des médicamens» ${ }^{29}$. Consecuentemente, defendía la necesidad «d'une étude sérieuse et profonde de la chimie» para una adecuada práctica de la farmacia $y$, al mismo tiempo, para favorecer que los farmacéuticos pudieran seguir realizando contribuciones destacadas a la química como hasta la fecha ${ }^{30}$. También Carbonell i Bravo, traductor de este escrito, recordaba el papel que habían jugado los farmacéuticos españoles en el avance de la química:

«si recorremos los progresos y adelantamientos de la ciencia química en España, si exâminamos con atencion el modo cómo se ha propagado en nuestras

27 Fourcroy, A. F.: «Discours sur l'union de la chimie et de la pharmacie, prononcé à la Société libre des pharmaciens de Paris, le 16 nivose an V de la République», Annales de chimie, 21 (1797),294-333. La traducción apareció como FOURCROY, A. Discurso sobre la unión de la química y la farmacia, traducido del francés por el Dr. D. Francisco Carbonell y Bravo, Madrid, Repullés, 1804.

28 FOUCROY (1797), op. cit. (27), pp. 306-307.

29 Ibid. p. 313.

30 Ibid. 319. Fourcroy recordaba que «la pharmacie ne tireroit aucun véritable avantage d'une étude légère et superficielle de la chimie, de même que ceux qui se seroient bornés à cette surface, ne pourroient pas rendre à la chimie, dans leurs opérations pharmaceutiques, les services qu'elle a droit d'attendre d'eux».

Hispania, LXI/2, núm. 208 (2001) 539-562 
provincias, nos convenceremos fácilmente que este trabajo ha sido en gran parte el fruto de la aplicación y desvelos de algunos farmacéuticos»31

Buenos representantes de este grupo de farmacéuticos, que habían contribuido al avance de la química, eran Gutiérrez Bueno y su discípulo y ayudante de cátedra en el Colegio de Farmacia, Antonio de la Cruz y Martín (17611817). Este último, que asistió a los primeros cursos de Gutiérrez Bueno en el Real Laboratorio de Química, ganó, en 1788, un primer premio en los concursos públicos realizados por los practicantes de farmacia de los Reales Hospitales de Madrid. En su disertación, Antonio de la Cruz defendió «lo mucho que influye la Química en todos los asuntos de la Farmacia», y, particularmente, en el tema que presentó: «la manera de hacer esencias, extraer el principio oloroso de los cuerpos, y modo de hacer las aguas de olor espirituosas y no espirituosas». Este joven farmacéutico se excusaba, en marzo de 1788, sólo un año después de la aparición del Méthode de nomenclature chimique, por «no explicarse por la nueva nomenclatura química». Aunque Antonio de la Cruz afirmaba que no le hubiera sido «muy difícil» emplearla, consideraba que no estaba "aún muy extendida» y que resultaba más conveniente emplear denominaciones «más generales y comprehensibles» ${ }^{32}$. Este comentario, así como las citas que Antonio de la Cruz realizó en su ejercicio de autores como Boerhaave y Macquer, muestran su buen conocimiento de la química del siglo XVIII, el cual debió mejorar en los cursos de Gutiérrez Bueno. A través de estos cursos, Antonio de la Cruz pudo conocer la obra de Antoine Fourcroy y de otros autores que habían tratado de aplicar la nueva química a la medicina y a la farmacia. En los años siguientes, este farmacéutico tradujo algunas obras destacadas relacionadas con esta materia, entre las que figuraba el estudio de John Rollo sobre la diabetes, en la versión comentada por Fourcroy, y el ensayo del discípulo de Fourcroy, Pierre-Philippe Alyon, sobre las propiedades médicas del oxígeno ${ }^{33}$. Asimismo, tradujo el «Tratado de los medios de desinfectar el ayre» de Guyton de Morveau y una obra de Luigi Vincenzo Brugnatelli, que incluía un texto de Fourcroy sobre toxicología ${ }^{34}$. A principios del siglo XIX, Antonio de la Cruz

31 FOURCROY (1804), op. cit. (27), pp. 32-33.

32 Memorial literario, 286 (junio de 1788), 289-290, «Noticia de los exercicios públicos que hacen anualmente los Practicantes de Farmacia de los Reales Hospitales Generales de esta corte, aspirando a los premios que ofrece la Real Junta a ellos».

33 ALYON, P.: Ensayo sobre las propiedades medicinales del Oxígeno ... Madrid, Pantaleón Aznar., 1798; Rollo, J.: Tratado del Diabetes Sacarino ... por ... Traducida del inglés, con varias notas del Ciudadano Fourcroy ... por el ciudadano Alyon ... y al castellano por D. Antonio de la Cruz. Madrid, Aznar, 1799; Sobre estas obras, v. HOLMES, F.L., "The chemical revolution and the art of healing", Caduceus, 11 (2) (1995), 103-126, especialmente, pp. 116-118.

34 GUYTON DE MORVEAU, L.: Tratado de los medios de desinfectar el ayre, precaver el contagio, y detener sus progresos. De ... Traducido de D. ... Madrid, Imprenta Real, 1803; BRUGNATELLI, L. Discurso sobre la acción de los medicamentos en el cuerpo humano ... Madrid, Imprenta de González, 1798. 
planeaba publicar una obra sobre «las experiencias hechas en España con los remedios oxîgenantes»:

«Creo que no tardaré en publicar las experiencias hechas en España con los remedios oxîgenantes, tales como ácido nítrico, cítrico, etc.; tengo buen número de ellas ya, y entre estas las hechas por dirección de Don Antonio Iasi, ProtoMédico del Exército del acantonamiento de Gibraltar, las que están acordes con mi opinión, igualmente que con las de muchos facultativos de esta Corte; ... Todo facultativo que hubiere hecho alguna, y quisiere en beneficio de la humanidad comunicármela, la publicaré, no deteniéndose en que los resultados hayan sido negativos; pues varias veces abren estos un camino nuevo a la observación» 35

Este proyecto de Antonio de la Cruz muestra su vinculación con las ideas de Fourcroy, quien, como es sabido, realizó numerosos trabajos para investigar las propiedades terapéuticas del oxígeno. Otro alumno de los cursos de Gutiérrez Bueno colaboró en la elaboración de una versión castellana de una revista que Fourcroy publicó en la última década del siglo XVIII bajo el título de $L a$ médécine éclairée par les sciences physiques ${ }^{36}$. Se trata de José Garriga (n. 1763), un abogado de los Reales Consejos que es más conocido por sus aportaciones posteriores a la meteorología y la astronomía ${ }^{37}$. Como el título de la revista indica, el objetivo de Fourcroy consistía en «prouver l'utilité de toutes les Sciences

Incluye abundantes notas de Antonio de la Cruz así como una traducción del Discurso sobre los contravenenos y en particular contra los acres y corrosivos por el C. Fourcroy en las pp. 86-115.

35 GUYTON DE MORVEAU (1803), op. cit. (34), p. 1, nota 1. Un resumen de los trabajos y opiniones de Fourcroy se encuentra en FOURCROY, A.: «Sur l'application de la chimie pneumatique à l'art de guérir, et sur les propriétés médicamenteuses des substances oxigénées», Annales de Chimie, 28, 225-279 (1799), texto resumido y comentado por Guyton de Morveau en la obra traducida por Antonio de la Cruz. Cf. GuYTON DE MORveau (1803), op. cit. (34), 182-183. Fourcroy también dejó sin publicar un libro que pretendía titular Oxigénologie. V. SMEATON, W. A.: Fourcroy chemist and revolutionary, Cambridge, University Press, 1962, pp. 207-209 y HOLMES, F. L. (1995), op. cit. (33), pp. 111-114.

${ }_{36}$ La Médecine éclairée par les sciences physiques, ou Journal des découvertes relatives aux différentes parties de lart de guérir, rédigé par M. Fourcroy, París. Los dos únicos volúmenes aparecieron entre 1791 y 1792. La traducción castellana apareció poco después bajo el título de «Diario de los nuevos descubrimientos de todas las Ciencias Físicas, que tienen alguna relación con las diferentes partes del arte de curar ...", Madrid, Sancha, 1792-93, 2 vols.

37 José Garriga había participado en un ejercicio público realizado al principio de las clase de Gutiérrez Bueno: Exercicio Publico de Quimica que tendran en el Real Laboratorio de esta corte Don Pasqual Arbuxech, Don Mariano Martinez Galinsoga, Don Josef Garriga, Don Francisco Campuzano. Baxo la direccion de D. Pedro Gutiérrez Bueno... Madrid, Antonio de Sancha, 1788. Cf. GaGo, R.: «The New Chemistry in Spain», Osiris, 1988, 4, 169-192, p. 180. Sobre la formación de este personaje, que fue más adelante conocido por su labor en el campo de la astronomía, v. Relación de los méritos, títulos, grados y exercicios literarios del licenciado D. Josef Garriga, abogado de los Reales Consejos y del Ilustre Colegio de esta Corte, Madrid, 27 de febrero de 1791. Sobre las actividades posteriores de este poco conocido personaje, v. Tinoco, J.: Apuntes para la bistoria del Observatorio de Madrid, Madrid, 1951, pp. 1516. 
Physiques pour la Médecine» recordando que «l'art de guérir ne peut faire de véritables progrès et avancer vers sa perfection que par le secours de toutes les Sciences réunies» 38 . Para defender la necesidad de su difusión en España, los traductores de esta revista atacaron en su introducción dos ideas que consideraban muy difundidas entre los médicos españoles. En primer lugar, criticaban la creencia de que «la Medicina práctica está fundada únicamente en la experiencia y observación, y que estas sólo se aprenden a la cabecera del enfermo» así como la idea asociada de «que la Física, la Chîmica, la Anatomía, etc. no sirven más que para forjar vanos sistemas». También censuraban «a ciertos profesores» que afirmaban "que cada país requiere su medicina particular» y que, por ello, consideraban «inútiles todos los conocimientos médicos sacados de las observaciones, y experiencia de otras naciones" ${ }^{39}$. Estos comentarios muestran que la enseñanza de la química desarrollada por Gutiérrez Bueno debía ser objeto de críticas por parte de algunos médicos, además de las ya senaladas entre los boticarios.

No obstante, las nuevas enseñanzas gozaban con el apoyo de instituciones como la Real Academia Médica Matritense, lo que no resulta sorprendente teniendo en cuenta que dentro de ella existían secciones dedicadas a las ciencias naturales y que uno de sus objetivos era «el adelantamiento de la Medicina en todas sus partes por medio de la experiencia y la razón», para lo que pretendían «aprovecharse de las muchas y grandes utilidades que le proporcionan las $\mathrm{Ma}$ temáticas, especialmente las mixtas, [...] la Física Experimental, Chîmica, Botánica y demás ramos de la Historia Natural» ${ }^{40}$. En su censura de la traducción de la revista de Fourcroy, los miembros de la Academia Médica Matritense destacaban la gran importancia de esta obra para difundir rápidamente «todos los descubrimientos al paso que se vayan haciendo [...] no sólo en la Medicina sino también en sus diferentes ramos y en las ciencias naturales que tienen relación con ella»:

«En España una obra de esta naturaleza en lengua vulgar es, digámoslo así, absolutamente indispensable por carecer el común de la Nación, de la noticia de los progresos que hacen las otras, que se tienen por cultas, y ser acreedora a que se le comuniquen con tal que esto se haga sin perjuicio de la religión verdadera, de las buenas costumbres y del buen orden y tranquilidad .... ${ }^{41}$.

38 FOURCROY, A.F.: «Introduction a...», La médecine éclairée par les sciences physiques..., 1 (1791), 3-47, en pp. 6-7.

39 Diario de los nuevos descubrimientos de todas las ciencias físicas, que tienen relación con las diferentes partes del arte de curar. Publicado en París por M. Fourcroy, Madrid, Imprenta de Sancha, 1792-1793, vol. I, pp. v-vi y viii.

40 Real Cédula por la que S.M. manda la observancia de los estatutos insertos de la Real Academia Médica de Madrid, Aranjuez, 23 de mayo de 1796. Véase, además, Noticia bistórica del origen, progresos y estado actual de la Real Médica de Madrid... Madrid [ca. 1796].

41 Archivo Histórico Nacional (AHN), Consejos, Legajo 5557, Informe de la Real Academia Médica Matritense, 26 de mayo de 1791.

Hispania, LXI/2, núm. 208 (2001) 539-562 
Las citas anteriores muestran que la aplicación de la química a la medicina durante los años de finales del siglo XVIII era un objeto de debate entre médicos y farmacéuticos. Hemos comprobado que uno de los principales protagonistas de esta polémica fue Antoine Fourcroy. A través de su revista y otros escritos, Fourcroy defendió la necesidad de integrar la química en la formación de médicos y farmacéuticos, actividad que impulsó desde su cátedra en la nueva Ecole de Santé de París creada en 1794. También difundió una nueva imagen de las relaciones entre la química y la farmacia, en la que esta última era considerada un arte que sólo podía superar sus prácticas rutinarias a través de un íntimo contacto con la nueva química. Todo ello llevaba aparejado una reforma de la tradicional formación de los boticarios y el establecimiento de nuevas instituciones donde pudieran recibir una educación académica en ciencias como la química.

\section{Las opiniones de Pedro Gutiérrez Bueno sobre las Relaciones ENTRE LA QUÍMICA Y LA FARMACIA.}

Dado que Gutiérrez Bueno y sus discípulos fueron uno de los principales introductores en España de las ideas defendidas por Fourcroy, resulta fácil encontrar huellas de las mismas en el pensamiento del autor analizado, tanto en la estructura de sus textos como en sus posiciones respecto a la enseñanza de la química y en sus puntos de vista sobre las relaciones entre la química y la farmacia. Como ya ha sido mostrado en otro trabajo, Gutiérrez Bueno empleó los libros de texto de Fourcroy como modelo para escribir algunas de sus obras. Como Fourcroy, Gutiérrez Bueno defendió la necesidad de cursos de química para los aspirantes al ejercicio de la farmacia. También afirmó que tales cursos debían aunar la enseñanza teórica con la práctica de operaciones químicas.

En la primera edición de su libro de texto, Gutiérrez Bueno dedicó varias páginas a defender sus ideas epistemológicas acerca de la química criticando tanto la rutina empírica como la teoría que no tenía su fundamento en la experiencia. De este modo, Gutiérrez Bueno consideraba que «la química tiene la ventaja de que su teoría se aprende al mismo tiempo que la práctica, porque los hechos son los que la hacen estable y clara» ${ }^{42}$. Para que esta enseñanza fuera fructuosa, por lo tanto, el estudiante debía compaginar la lección teórica con las prácticas del laboratorio, tal y como defendió en su segunda edición de la obra:

«En vano se esfuerza para aprender esta ciencia el que no junta a la lectura y a la meditación la asistencia a un laboratorio en que observe y execute por sí las operaciones que se propone executar» ${ }^{43}$.

42 [GUTIERREZ BUENO] (1788), op. cit. (1), pp. 47-48.

43 Gutierrez Bueno (1802), op. cit. (1), p. 10. En la p. 262 de esta misma obra, señalaba también como que «Sin embargo, los mismos habrán advertido que, aunque en las lecciones impre-

Hispania, LXI/2, núm. 208 (2001) 539-562 
De acuerdo con estas ideas didácticas, Gutiérrez Bueno realizó numerosas experiencias públicas en sus cursos de química, gracias a las instalaciones que dispuso en el Real Laboratorio de Química de Madrid. Poco después de ser apartado de este laboratorio, que pasó a manos de Louis Proust, Gutiérrez Bueno describió en uno de sus escritos el modo en el que se desarrollaron sus enseñanzas:

«Mientras estuvo a mi cargo la enseñanza de la chîmica en el Real Laboratorio de la calle de Alcalá, no omití ningún tratado de cuantos pudiesen contribuir a completar la instrucción pública en todo lo que tiene relación con esta gran ciencia; y no contentaba con teorías, sino que por medio de repetidos experimentos hacía que los asistentes a las lecciones se convenciesen por sus sentidos de cuanto explicaba»44

Existen otras referencias que indican que Gutiérrez Bueno realizó ante sus alumnos operaciones químicas relacionadas con nuevos métodos para blanquear y teñir telas y con el análisis de aguas, algunas de las cuales describió en sus publicaciones ${ }^{45}$. Más adelante, cuando se encontraba al frente del Colegio de Farmacia de Madrid, defendió con firmeza la necesidad de un laboratorio adecuado para sus clases. En 1807, encabezó un escrito con otros profesores del Colegio, donde manifiestaba sus quejas por no tener «un laboratorio químicofarmacéutico tal como es necesario ... para la execución de las operaciones químicas y elaboraciones farmacéuticas con que se ha de enseñar a los discípulos como para las operaciones de práctica que se señalan por punto de examen a los que se revalidan ${ }^{46}$. Es importante tener en cuenta este último aspecto: los estudiantes de farmacia debían obtener su título a través de un examen práctico que incluía la elaboración de una preparación farmacéutica y, debido a ello, la formación práctica era una cuestión de gran importancia ${ }^{47}$. Hasta la aparición de los Colegios de Farmacia, esta formación había sido adquirida a través del aprendizaje en las boticas. Las nuevas instituciones educativas debían buscar nuevos modelos de enseñanza que permitieran adquirir estas habilidades prácticas.

Como otros profesores de finales del siglo XVIII, Gutiérrez Bueno consideraba que la asistencia a las clases teóricas y la observación de las experiencias que allí se realizaban no era suficientes para el correcto aprendizaje de la quí-

\footnotetext{
sas se insisnuan sólo muchas cosas, o se tocan ligeramente, no les habrá quedado nada que desear para su instrucción en la explicación extendida de cada una, a que siempre se ha acompañado la parte práctica que es la que más convence al entendimiento, y la que se mira como principal en esta importante ciencia».

44 Gutierrez Bueno, P.: Análisis de las aguas de Madrid. Por Don ..., chîmico de esta corte según se balla en el Semanario de Agricultura y Artes. Madrid, Villalpando, 1800, p. 21.

45 V. Garcia Belmar, A.: (en publicación) y el resto de obras citadas en la nota 2.

46 AMHF, Libro de actas del Colegio de Farmacia, f. 60, Junta ordinaria de 14 de enero de 1807.

47 Para algunos ejemplos de los contenidos de estos exámenes, v. AMHF, Libro de exámenes de Farmacia celebrados en este Real Colegio de Madrid de la misma facultad.
} 
mica. También era necesario que los estudiantes repitieran las operaciones químicas y realizaran personalmete algunos experimentos. Para ello, publicó un volumen dedicado a la parte «Práctica del Curso de Química» donde ofrecía descripciones detalladas de un gran número de experiencias, con el objetivo de que sus lectores pudieran «repetir por sí todos los experimentos, asegurarse de ellos, y adelantar en los mismos conforme a su talento, luces y aplicación» ${ }^{48}$. Aunque tales descripciones detalladas son frecuentes en los libros de texto de química de esos años, la publicación de un volumen particular dedicado a esta cuestión es una característica que distingue la obra de Gutiérrez Bueno de la mayor parte de otras obras semejantes contemporáneas. Para comprender esta característica singular, resulta necesario repasar las ideas didácticas que defendió Gutiérrez Bueno y el marco en el que se produjo la publicación. En varios textos, Gutiérrez Bueno afirmó la necesidad de que los estudiantes de química realizaran experimentos «con instrumentos exactos y construidos por sí o por su dirección» ${ }^{49}$. Estos experimentos sencillos y fácilmente reproducibles, que un gran número de personas podían realizar, figuran en obras semejantes publicadas en otros países europeos en esos años. Entre ellas figuran las realizadas por Friedrich Accum que estaban dirigidas a un público amplio interesado por reproducir personalmente algunos de los más recientes experimentos de la química, lo que generó un comercio de instrumentos y productos químicos del que se beneficiaron los autores de estas obras ${ }^{50}$.

Es posible que la obra de Gutiérrez Bueno estuviera también dirigida a un público semejante. El número de experiencias que recogía era muy elevado y resulta difícil que todas ellas fueran realizadas durante su curso en el Colegio de Cirugía de San Carlos o en otras instituciones donde impartió clases. Por otra parte, el texto contenía tanto descripciones de la preparación de ciertas sustancias, semejantes a las recetas que aparecían en los libros de farmacia, como experiencias destinadas a servir como demostraciones experimentales de las afirmaciones que se realizaban en la teoría. Estas últimas podían presentar más interés para "personas libres de las preocupaciones comunes» interesadas en la química, como las que acudieron durante los años finales del siglo XVIII a sus cursos en el Real Laboratorio de Química de Madrid. Gutiérrez Bueno realizó cursos privados de química destinados, en parte, a este nuevo público. Así consta en las declaraciones de los testigos que certificaron su buena conducta durante los

48 Gutierrez Bueno (1802), op. cit. (1), p. 6. Al final del curso, los estudiantes deberían estar en disposición de «executar las demás [operaciones] que practican los profesores de química». $C f$. GUTIERREZ BUENO (1803), pp. 1-2.

49 Gutierrez Bueno (1803), op. cit. (1), p. 61.

50 Sobre esta cuestión, en el caso de Inglaterra y las obras de F. Accum, v. DolAN, B.: «The Language of Experiment in Chemical Textbooks: Some Examples from Early Nineteenth-Century Britain». En: A. LundGren; B. BENSAUde-VInCENT (eds.): Communicating Chemistry. Textbooks and Their Audiences, 1789-1939, Canton, Science History Pub., 2000; GolInSKI, J.: Science as Public Culture: Chemistry and Enlightenment in Britain, 1760-1820, Cambridge, University Press, 1992, pp. 129-151. 
años del gobierno afrancesado. A pesar de que se encontraba enfermo, durante los años de ocupación francesa, Gutiérrez Bueno realizó lecciones paticulares en su casa, a las que concurrían "profesores y aficionados a las ciencias físicas», entre ellos algunos «boticarios» y «oficiales franceses», así como «otros aficionados a las ciencias de Química y Farmacia», que gozaban con el «recreo y distraimiento que lleva consigo el discurso y la controversia sobre dichas ciencias» ${ }^{51}$. De este modo, con esta "parte práctica» de su curso de química, que inducía a sus lectores a «repetir por sí todos los experimentos», Gutiérrez Bueno estaba fomentando un pequeño mercado de instrumentos y productos químicos generado por todos aquellos interesados en realizar sencillas experiencias químicas, bien como complemento de su formación o bien, simplemente, como un pasatiempo o divertimento ${ }^{52}$.

A pesar de esta clara orientación práctica de sus enseñanzas, no conviene olvidar que Pedro Gutiérrez Bueno también defendía la necesidad del aprendizaje de ciertas nociones teóricas que debían guiar las experiencias y permitir la renovación de las prácticas rutinarias:

«La experiencia que no es dirigida por la teoría, será siempre un tacto de ciego que le hace perder el tino en saliendo del camino trillado. Por el contrario, la teoría sin la experiencia nunca será otra cosa que un resplandor, que en vez de dar luz, deslumbres3»

En un interesente pasaje de una de sus obras sobre análisis de aguas, este farmacéutico diferenciaba los requisitos necesarios para estudiar la química «como ciencia o como arte», señalando las ventajas del primer tipo de aprendizaje frente al segundo. Este interesante párrafo nos informa, además, de las ideas de Gutiérrez Bueno con otras disciplinas cercanas como la física experimental o la minerometalurgia:

«La chîmica se puede considerar como ciencia o como arte; el que emprenda su estudio, como ciencia, ha de tener buenos principios de matemáticas: con ellos entrará a estudiar la física experimental, la botánica, la mineralogía, metalurgia, y en general la historia natural: rodeado de estas ciencias auxiliares ha de leer y operar con reflexión, aplicando a sus experimentos los conocimientos que ha adquirido en cada una de ellas: por exemplo, si quiere ensayar un agua, la física le

51 Archivo del Palacio Real (APR), Caja 490, Exp. 26. Información judicial justificando D. Pedro Gutiérrez Bueno su conducta patriótica durante la dominación de los Franceses en Madrid, 1814. Las citas proceden de las declaraciones de los testigos D. Julián Melón, presbítero, y D. Pedro Muro, agente de negocios. Gutiérrez Bueno también defendía que a sus clases habían concurrido «diferentes Profesores y aficionados a las ciencias físicas» con el fin de disfrutar del «recreo y distraimiento que lleva consigo el discurso y la controversia sobre dichas ciencias». Cf. Ibid. Carta de Pedro Gutiérrez Bueno, Madrid, 13 de julio de 1814.

52 La cita anterior procede de P. GUTIERREZ BUENo (1802), op. cit. (1), p. 6

s3 [GuTIERREZ BuENO] (1788), op. cit. (1), pp. 47-48. 
enseñará cuáles son las propiedades generales de los fluidos, el modo de saber su peso absoluto y específico, densidad, temperatura, etc.; la mineralogía y metalurgia, la naturaleza de los precipitados que ocasionen los reactivos; y sin el auxilio de las matemáticas no podrá determinar la figura de las sales, ni conocerlas, ni formar cálculos de los cuerpos sólidos, líquidos y aeriformes que resulten.

Pero para el que solo hace de la chîmica un arte, basta que tenga libros, que repita las operaciones, procediendo en ellas conforme a las fórmulas de cada una, y siempre hallará alguna cosa que notar; pero sólo debe hacer méritos de aquellas que sean de alguna utilidad. El científico nunca hallará dificultades; el mero operador tropezará a cada instante, y se verá muchas veces engañado por sus ojos y por su imaginación ${ }^{54}$ »

En este párrafo, aparece la diferenciación entre «ciencia» y «arte» que había sido empleada por muchos autores del siglo XVIII para discutir las características de la química. En los años en los que Gutiérrez Bueno escribía, estas categorías estaban siendo reemplazadas por las de «química pura» y «química aplicada» que fueron difundidas por autores como el sueco Johann Gottschalk Wallerius, tal y como ha sido señalado por Christoph Meinel55. Gutiérrez Bueno defendió en varios de sus escritos las aplicaciones de la química a varias actividades, entre ellas el «arte de curar», y empleó estos argumentos para defender la utilidad de sus enseñanzas, de acuerdo con las tendencias predominantes durante la Ilustración ${ }^{56}$. Empleando la diferenciación de la cita anterior, resulta evidente que Gutiérrez Bueno defendía la necesidad del estudio de la «química como ciencia» para todos aquellos que pretendían dedicarse al «arte de curar». En el prólogo de su libro dirigido a los estudiantes del colegio de cirugía de Madrid, afirmaba que sus estudiantes debían tener previamente «buenos principios de matemáticas, de física experimental, mineralogía, y, en general, de historia natural» y dedicarse la química «con la mayor aplicación por espacio de dos años, que es lo menos que necesita un hombre de talento para poder hablar con algún acierto de lo que otros hacen o escriben" ${ }^{57}$. Estas materias formaban también parte de los requisitos que permitían la admisión de los estudiantes en los colegios de Farmacia donde impartió clases Gutiérrez Bueno. En realidad, tales exigencias formativas eran difícilmente compatibles con la tradicional formación empírica de cirujanos y de boticarios, desarrollada en el marco de los

54 Gutierrez Bueno (1800), op. cit. (44), pp. 22-23.

55 MeInel, C.: «De praestantia et utilitate Chemiae. Selbstdarstellung einer jungen Disziplin im Spiegel ihers programmatischen Schrifttums", Sudhoffs Arcbiv, 65 (1981), 366-389. MEINEL, C.: "Theory or practice? The eightennth-century debate on the scientific status of chemistry", Ambix, 30 (1983), 121-132.

56 Garcia Belmar (2000), op. cit. (2).

57 Gutierrez Bueno (1802), op. cit. (1), p. 2. V. también ibid. pp. 97-98 donde señala que aquellos que se dedicasen al arte de curar debían «estudiar la química con particular cuidado a lo menos dos años para sacar de esta ciencia nociones muy importantes a su profesión».

Hispania, LXI/2, núm. 208 (2001) 539-562 
gremios. Por ello, los requisitos exigidos por Gutiérrez Bueno suponían la existencia de nuevas instituciones educativas.

Desde esta perspectiva, la enseñanza de la química, tal y como la concebían autores como Gutiérrez Bueno, quedaba íntimamente ligada a las reformas institucionales de principios del siglo XIX que transformaron las diferentes ocupaciones sanitarias. En una dedicatoria, Gutiérrez Bueno alababa la labor del Príncipe de la Paz cuyas reformas habían impulsado la introducción de la enseñanza de las «ciencias auxiliares» en la formación de las aspirantes a diversos títulos relacionados con la medicina y la farmacia:

«Al Excmo. Señor Príncipe de la Paz: «Como V.E. fue el primero que en España ha sabido reunir con más acierto el estudio de las ciencias auxîliares al de aquel arte tan importante que tiene por objeto la conservación de la salud y el alivio de la doliente humanidad espero que se dignará recibir con la benignidad que le caracteriza la breve noticia que publico de los primeros experimentos sobre el Galvanismo nuevamente descubierto, en cuyas propiedades particulares se fundan grandes esperanzas para los progresos de la medicina» 58

Este folleto dedicado al galvanismo comenzaba con unas breves referencias históricas a los trabajos de Galvani seguidas por algunas nociones teóricas previas al grueso de la obra: la descripción detallada de más de veintitrés experiencias. Las primeras experiencias estaban destinadas a presentar los sorprendentes efectos de este «fluido galvánico» en ranas muertas mientra que en la segunda parte estaba consagrada a analizar sus efectos sobre el cuerpo humano. La última, y la más extensa de todas las experiencias, incluía indicaciones muy precisas respecto al uso de este fluido para la curación de «las enfermedades de ojos, sorderas y parálisis» $"$ ?

Este folleto muestra claramente que, a finales del siglo XVIII, la defensa de la utilidad de la química para el «arte de curar» era un buen argumento para aquéllos que, como Pedro Gutiérrez Bueno, impulsaron la creación de las nuevas instituciones educativas. Por ello, no resultan sorprendentes las constantes referencias a estas cuestiones en otros libros de Gutiérrez Bueno. En la primera edición de su libro de texto, dedicó la mayor parte de su introducción a describir los usos de la química para la producción de medicamentos y de contravenenos, la separación de «las partes en que reside la virtud de ciertas plantas» y las investigaciones toxicológicas ${ }^{60}$. Del mismo modo, en la edición de 1802,

58 GUTIERREZ BUENO, P.: Observaciones sobre el galvanismo, según se ballan en el Curso de la práctica química de D. ... con la descripción de los aparatos y modo de usarlos. Madrid, Villalpando, 1803, pp. I-II: «Al Excmo Señor Príncipe de la Paz: "Como V.E. fué el primero que en España ha sabido reunir con más acierto el estudio de las ciencias auxîliares al de aquel arte tan importante que tiene por objeto la conservación de la salud y el alivio de la doliente humanidad ...»

59 Ibid. pp. 25-30.

60 [Gutierrez Bueno] (1788), op. cit. (1), p. 19. V. Garcia Belmar (2000), op. cit. (2). 
afirmaba que la química era «la ciencia» de la que debía «esperar el género humano el auxilio más importante, cual es la conservación de nuestra salud» ${ }^{61}$. A lo largo de esta segunda edición, en la descripción de las diferentes sustancias, Gutiérrez Bueno ofreció numerosos ejemplos de estas aplicaciones médicas de la química, con especial atención a las cuestiones relacionadas con la preparación de medicamentos. Por ejemplo, al final del capítulo dedicado a los óxidos, Gutiérrez Bueno señalaba la utilidad de estos conocimientos para el arte de los tintoreros y para aquéllos «que se dedican al arte de curar». Según Gutiérrez Bueno, estos últimos debían «saber el estado y gravedad absoluta de oxígeno que tiene cada uno de los cuerpos quemados" y, como ejemplo, ofrecía el caso de «los oxîdes de antimonio»:

«Los oxîdes de antimonio, que se miran en la medicina como uno de los principales medicamentos, apenas se preparan con uniformidad por los profesores de farmacia. Los médicos y cirujanos piden la dosis que necesitan, y sino está bien preparada, el enfermo es el único que lo padece. Si puede causar graves daños la mala preparación de este solo oxîde ¿Qué será de la de todos los demás medicamentos? por eso es muy debido que los que hayan de curar estudien esta ciencia, y sepan conocer la calidad de las medicinas, y no proceder a ciegas fiados en la buena fe de los farmacéuticos» ${ }^{62}$.

En otros apartados, Gutiérrez Bueno empleó los conocimientos químicos para poner en cuestión las virtudes terapéuticas atribuidas a ciertas sustancias. En el capítulo dedicado a la "tierra alumine o arcilla pura», señalaba los usos terapéuticos de productos tradicionalmente empleados en farmacia como el «bolo arménico», «la tierra sellada o lemnia» o «la piedra de cananor». También criticaba las "virtudes extraordinarias» atribuidas especialmente a estas dos últimas, que incluían la curación de la peste y de casi cualquier otra enfermedad, y recordaba que «el químico sólo debe conocer que su virtud consiste en ser un medicamento astringente por la tendencia que tiene el alúmine a combinarse con el agua y con los ácidos en el estómago» ${ }^{63}$. De nuevo seguía Gutiérrez Bueno las ideas de Fourcroy que, en esos años, defendía el uso de la química para la reforma de la terapéutica y, en particular, para desvelar las supuestas virtudes de los "remedes mystérieux». El autor francés consideraba que el análisis químico debía ser capaz de «éclairer sans cesse le public, sur les recettes pillés dans les anciennes pharmacologies» diferenciando entre «ce qui sera utile et digne d'être conservé, d'avec ce qui n'appartiendra qu'à l'ignorance

61 Gutierrez BuENO (1802), op. cit. (1), p. 3.

62 Ibid. pp. 97-98.

63 Ibid, pp. 131-132. Otro ejemplo, v. ibid. p. 133-134. Estas críticas aparecen ya en obras anteriores. Cf. Félix Palacios, Palestra pharmaceutica, chymico-galenica..., Madrid, Juan García, 1706, pp. 21 y 679 . 
ou à la charlatanerie» ${ }^{64}$. Estas ideas formaban parte de la nueva actitud crítica hacia los remedios secretos que se desarrolló durante el siglo XVIII ${ }^{65}$.

\section{CONCLUSIONES}

Gutiérrez Bueno asistió durante sus años de profesor a importantes transformaciones en las relaciones entre la química y la farmacia. Hemos comprobado que sus puntos de vista sobre este asunto presentan grandes similitudes con los defendidos por Antoine Fourcroy en Francia, autor que también inspiró las nuevas instituciones de enseñanza de la farmacia surgidas a principios del siglo XIX que sirvieron de modelo para el establecimiento de los Colegios de Farmacia de Madrid y de Barcelona. Las obras de Fourcroy fueron empleadas por Gutiérrez Bueno para estructurar y redactar apartados de su obra, tal y como hicieron otros autores españoles de esos años. Hemos comprobado la labor realizada por Gutiérrez Bueno y algunos de sus discípulos, como Antonio de la Cruz o José Garriga, en la asimilación de las ideas de este autor francés, tanto a través de traducciones como de sus propias obras, lo que contribuyó a asegurarle un importante papel en la química española de los años finales del siglo XVIII. La asimilación de la obra de este autor, realizada por Gutiérrez Bueno y sus discípulos, sirvió no sólo para promover el triunfo de las nuevas ideas generalmente asociadas a la "revolución química», sino también para difundir nuevas imágenes sobre las relaciones disciplinares entre la química y la farmacia.

Estas nuevas imágenes disciplinares, que transformaban a la química en una ciencia fundamental para el avance de la farmacia, estaban íntimamente ligadas con la reforma de la enseñanza y el ejercicio de la farmacia que ocurrieron a finales del siglo XVIII. Al defender la necesidad del estudio de la química para los farmacéuticos, de una nueva química con fuertes relaciones con la física experimental, Gutiérrez Bueno estaba también impulsando la desaparición progresiva del antiguo sistema de enseñanza basado en el aprendizaje en las boticas y controlado por los gremios de farmacéuticos. Esta transformación se realizó con fuertes resistencias de algunos boticarios que no consideraban necesaria la asistencia de sus ayudantes a las clases de los nuevos Colegios de Farmacia. Los partidarios de las nuevas instituciones educativas consideraban que este viejo sistema difícilmente podía ser compatible con la formación reglada en las ciencias naturales que debían contribuir - como aspiraba Fourcroy- a superar las prácticas rutinarias de los boticarios y, en definitiva, a la reforma de la terapéutica. Todo ello, exigía la creación de nuevas instituciones como los Colegios de Farmacia, tarea en la que Gutiérrez Bueno participó activamente, defendiendo, entre otras cuestiones, la necesidad de una dotación suficiente

64 FOURCROY (1797), op. cit. (27), p. 330.

6s Ackerknecht, E. H.: Therapeutics. From the Primitives to the 20th Century..., New York, MacMillan, 1973, pp. 89-90.

Hispania, LXI/2, núm. 208 (2001) 539-562 
para los laboratorios donde debía ofrecerse una adecuada enseñanza teórica y práctica de la química. Esta fue una de las causas que originaron su disputa con la Junta Gubernativa que dirigía el colegio de Farmacia y sufragaba sus gastos a través de los cuantiosos ingresos que proporcionaba la expedición de los diferentes títulos de farmacéuticos y las visitas a las boticas.

Gutiérrez Bueno defendió que la enseñanza de la química debía aunar la teoría con la práctica en los laboratorios. En consonancia con las ideas de Fourcroy, los aspectos teóricos debían servir para guiar las experiencias y evitar la rutina ciega cuyo único fundamento era la tradición. De este modo, Gutiérrez Bueno defendió una nuevo tipo de enseñanza práctica, no sólo enfocada a la preparación de ciertos productos farmacéuticos y al conocimiento de ciertas operaciones, sino también dirigida a la ilustración de las afirmaciones defendidas en la parte teórica. Tal y como hemos visto, Gutiérrez Bueno pretendía difundir experimentos sencillos y fácilmente reproducibles que pudieran ser realizados por los lectores de sus obras, con el fin de comprobar personalmente las afirmaciones recogidas en la parte teórica. Estos experimentos estaban probablemente dirigidos también a algunos «aficionados a las ciencias físicas» interesados en realizar pequeñas experiencias químicas como entretenimiento o diversión.

La defensa de Gutiérrez Bueno de la enseñanza práctica estaba relacionada con sus ideas acerca de las fuentes y los contenidos de los libros de texto. Gutiérrez Bueno se excusó en numerosas ocasiones por no incluir referencias a ciertas sustancias descritas por otros autores, debido a que no había podido repetir las correspondientes experiencias en su laboratorio. Estos comentarios no sólo reflejan los puntos de vista del autor estudiado sobre la relación entre teoría y experiencia sino que también recuerdan las grandes transformaciones que se produjeron en las características de los libros de texto y otros géneros de literatura científica durante el siglo XIX. A principios de esa centuria, autores como Gutiérrez Bueno todavía continuaban incluyendo observaciones originales en sus libros de texto, las cuales podían ser publicadas por primera vez en este tipo de obras. Esta práctica era habitual durante el siglo XVIII y fue poco a poco desapareciendo en el siglo XIX con la consolidación del libro de texto como un género de literatura científica con unas características diferentes a las que había tenido hasta ese momento.

El análisis anterior muestra que los cambios en los públicos destinatarios de los libros de texto de química fueron decisivos en el proceso de consolidación de estas obra como género de literatura científica. Muchas de sus características se transformaron para dar una respuesta adecuada a los intereses de sus nuevos lectores y para adaptarse a los nuevos espacios institucionales en los que se desarrollaron las clases. De este modo, pueden comprenderse mejor algunas de las principales transformaciones que Gutiérrez Bueno introdujo de la primera a la segunda edición de su libro de texto. No obstante, otros cambios introducidos por Gutiérrez Bueno sólo pueden ser comprendidos mediante el análisis de su actitud frente al proceso que suele designarse con el nombre de «revolución química». Esta perspectiva de análisis será desarrollada en un próximo artículo.

Hispania, LXI/2, núm. 208 (2001) 539-562 